\title{
Co-delivery of ccl19 gene enhances anti-caries DNA vaccine pCIA-P immunogenicity in mice by increasing dendritic cell migration to secondary lymphoid tissues
}

\author{
Yan-hong YAN ${ }^{1}$, Sheng-cai $\mathrm{QI}^{2}$, Ling-kai SU ${ }^{1}$, Qing-an $\mathrm{XU}^{1,3, *}$, Ming-wen $\mathrm{FAN}^{1, *}$ \\ ${ }^{1}$ The State Key Laboratory Breeding Base of Basic Science of Stomatology (Hubei-MOST) \& Key Laboratory of Oral Biomedicine of \\ Ministry of Education (KLOBM), School \& Hospital of Stomatology, Wuhan University, Wuhan 430079, China; ${ }^{2}$ Center of Stomatology, \\ Tongji Hospital, Tongji Medical College, Huazhong University of Science and Technology, Wuhan 430030, China; ${ }^{3}$ Department of Endo- \\ dontics, School and Hospital of Stomatology, Wuhan University, Wuhan 430079, China
}

Aim: To investigate how co-delivery of the gene encoding $\mathrm{C}-\mathrm{C}$ chemokine ligand-19 (CCL-19) affected the systemic immune responses to an anti-caries DNA vaccine pCIA-P in mice.

Methods: Plasmid encoding CCL19-GFP fusion protein (pCCL19/GFP) was constructed by inserting murine ccl19 gene into GFPexpressing vector pAcGFP1-N1. Chemotactic effect of the fusion protein on murine dendritic cells (DCs) was assessed in vitro and in vivo using transwell and flow cytometric analysis, respectively. BALB/c mice were administered anti-caries DNA vaccine pCIA-P plus pCCL19/GFP (each $100 \mu \mathrm{g}$, im) or pCIA-P alone. Serum level of anti-PAc IgG was assessed with ELISA. Splenocytes from the mice were stimulated with PAc protein for $48 \mathrm{~h}$, and IFN- $\gamma$ and IL-4 production was measured with ELISA. The presence of pCCL19/GFP in spleen and draining lymph nodes was assessed using PCR. The expression of pCCL19/GFP protein in these tissues was analyzed under microscope and with flow cytometry.

Results: The expression level of CCL19-GFP fusion protein was considerably increased $48 \mathrm{~h}$ after transfection of COS-7 cells with pCCL19/GFP plasmids. The fusion protein showed potent chemotactic activity on DCs in vitro. The level of serum PAc-specific IgG was significantly increased from 4 to 14 weeks in the mice vaccinated with pCIA-P plus pCCL19/GFP. Compared to mice vaccinated with pCIA-P alone, the splenocytes from mice vaccinated with pCIA-P plus pCCL19/GFP produced significantly higher level of IFN- $y$, but IL-4 production had no significant change. Following intromuscular co-delivery, pCCL19/GFP plasmid and fusion protein were detected in the spleen and draining lymph nodes. Administration of CCL19 gene in mice markedly increased the number of mature DCs in secondary lymphoid tissues.

Conclusion: CCL19 serves as an effective adjuvant for anti-caries DNA vaccine by inducing chemotactic migration of DCs to secondary lymphoid tissues.

Keywords: chemokine; CCL19; DNA vaccination; anti-caries DNA vaccine; dendritic cell; splenocyte; IFN-ץ; IL-4; stomatology

Acta Pharmacologica Sinica (2013) 34: 432-440; doi: 10.1038/aps.2012.153; published online 21 Jan 2013

\section{Introduction}

DNA vaccination is a promising strategy for inducing potent immune responses against a wide range of pathogens ${ }^{[1,2]}$. In one of our previous studies, it was demonstrated that a DNA vaccine pCIA-P encoding A-P region of pac gene of Streptococcus mutans (S mutans), a causative microorganism of dental

\footnotetext{
* To whom correspondence should be addressed.

E-mail fmw@whuss.com (Ming-Wen FAN); xuqingan1977@yahoo.com.cn (Qing-An XU)

Received 2012-06-17 Accepted 2012-09-29
}

caries, could elicit protective immunity in rodents via intramuscular immunization ${ }^{[3]}$. Although DNA vaccination often works well in small animal models, it displays poor efficiency in larger animals and human. Thus, it is a critical and urgent task to find ways to improve the immunogenicity of DNA vaccines. Following intramuscular injection of a plasmid DNA vaccine, the encoded gene is thought to be expressed in transfected myocytes at the site of inoculation ${ }^{[4]}$, as well as in a small number of dendritic cells (DCs) ${ }^{[5-8]}$. DCs capture the antigens expressed by transfected cells, process, and then present them to $\mathrm{T}$ cells in secondary lymphoid organs where naive 
$\mathrm{T}$ cells are activated ${ }^{[9]}$. In this process, DCs undergo radical changes from immature phagocytic precursor cells to potent antigen-presenting cells (APCs) and upregulate receptors for constitutive chemokines, such as $\mathrm{C}-\mathrm{C}$ chemokine receptor type 7 (CCR7) ${ }^{[10,11]}$. The magnitude of the immune responses against target antigens is strongly dependent on encounters between DCs and naive T cells. An enhanced number of antigen-presenting DCs in secondary lymphoid tissues leads to increased DC-T cell interaction, thereby facilitating immunity. Therefore, many recent efforts to improve the performance of DNA vaccines have been focused on attracting more DCs to lymphoid organs.

Chemokines play key roles in the regulation of immune responses, influencing effector cells of the innate and adaptive immune systems. Owing to their abilities to enhance or modulate immune responses induced by DNA vaccines, chemokines are receiving increasing attention ${ }^{[12]}$. Chemokine (C-C motif) ligand 19 (CCL19), the ligand of CCR7, is mainly expressed in stromal cells of the $\mathrm{T}$ cell zone in lymphoid organs ${ }^{[11,13,14]}$. CCR7 is expressed on mature DCs and distinct T and B cell subpopulations ${ }^{[11,13,14]}$. CCR7 and its ligand CCL19 are recognized as essential molecules for the directional migration of mature DCs and the establishment of a functional microenvironment to prime naive T cells ${ }^{[15-19]}$. During the past decades, several studies have demonstrated that codelivery of expression plasmids encoding CCL19 along with DNA vaccines led to the enhancement of humoral and $\mathrm{T}$ cell immune responses against viruses ${ }^{[20-23]}$ and tumors ${ }^{[24,25]}$. The enhanced immunogenicity was supposed to be derived from the exogenous overexpression of CCL19 in secondary lymphoid tissues, which might enhance DCs recruitment and increase the chance of an encounter between DCs and T cells ${ }^{[20,21,23]}$. However, until now, solid evidence indicating that an exogenously delivered CCL19 plasmid can enter secondary lymphoid tissues and express chemokine CCL19 has been lacking. To address this issue, we constructed a GFP-labeled plasmid pCCL19/GFP and investigated the existence and expression of pCCL19/ GFP in secondary lymphoid tissues after intramuscular administration in this study. Moreover, we assessed whether intramuscular pCCL19/GFP codelivery along with anti-caries DNA vaccine pCIA-P could elevate the efficiency of systemic immune responses and enhance $\mathrm{CD} 4^{+} \mathrm{T}$ cell mediated cytokine production. Additionally, we investigated the in vitro and in vivo chemotactic activity of pCCL19/GFP-expressed fusion protein on mature DCs. This study proposed a new approach for augmenting the immunogenicity of the anti-caries DNA vaccine and explained the underlying mechanism involved in the enhanced immune response.

\section{Materials and methods}

\section{Plasmid construction and preparation}

Murine ccl19 gene (NM 011888.2) was amplified from mouse spleen by RT-PCR with a TGA to ATG stop codon mutation. A pair of primers (forward: 5'-ATATAAGCTTATGGCCCCCCGTG-3', reverse: 5'-AAGGATCCATAGACACAGGGCTCC-3') was used to amplify the ccl19 gene. The ampli- fication product was inserted into the Hind III and BamH I sites of the GFP-expressing vector pAcGFP1-N1 (Clontech Laboratories, Inc, Mountain View, CA, USA) to obtain plasmid encoding CCL19-GFP fusion protein. The construct was confirmed by sequencing and designated as pCCL19/GFP. The DNA vaccine pCIA-P encoding A-P region of pac gene of $S$ mutans was used as an antigenic plasmid ${ }^{[3]}$. All plasmids were isolated and purified with the EndoFree Plasmid Giga kit (Qiagen, Germany) and stored at $-20^{\circ} \mathrm{C}$ until further use. Before inoculation, DNA plasmids were dissolved into endofree normal saline buffer to obtain a final DNA concentration of $1 \mu \mathrm{g} / \mu \mathrm{L}$.

\section{Plasmid transfection and in vitro expression analysis}

COS-7 cells (Chinese Center for Type Culture Collection, CCTCC, Wuhan, China) were transfected with pCCL19/ GFP using Sofast transfection reagent (Sunma Biotechnology Co, Ltd, Xiamen, China). The expression of the recombinant proteins labeled with GFP was observed under a fluorescence microscope. The supernatants were collected $48 \mathrm{~h}$ later and kept at $-80^{\circ} \mathrm{C}$. The recombinant protein secreted in the culture supernatant was analyzed using a mouse CCL19 ELISA kit (R\&D Systems, Minneapolis, MN, USA).

\section{Chemotaxis assays}

DCs were generated from murine bone marrow cells as previously described ${ }^{[26]}$. The cells were matured by incubating them with $100 \mathrm{ng} / \mathrm{mL}$ lipopolysaccharide (LPS, Sigma-Aldrich, St Louis, USA) for $24 \mathrm{~h}$. Surface marker expression (CCR7 and CD11c) was analyzed by flow cytometry. The cells were gated on CD11c ${ }^{+}$cells, and CCR7 expression levels were then analyzed. The chemotactic response of mouse DCs to CCL19GFP fusion proteins was detected by Transwell (5- $\mu \mathrm{m}$ pore size) in 24-well plates (Corning Costar, Cambridge, MA, USA). Briefly, the cultured supernatants from COS-7 cells transfected with the recombinant plasmid pCCL19/GFP or a blank vector pAcGFP1-N1 were added into the lower wells. Recombinant murine CCL19 peptide (100 ng/mL; Peprotech EC, London, UK) dissolved in the medium was used as a positive control. A total of $0.5 \times 10^{6}$ DCs in $200 \mu \mathrm{L}$ RPMI-1640 medium was added to the upper wells of the chemotaxis chambers. In some experiments, DCs were incubated with anti-mouse CCR7 antibody (eBiosciences, USA) for 30 min before being placed in the upper wells. Chemotaxis was allowed for $4 \mathrm{~h}$, and DCs that had migrated through the filter to the lower chambers were counted in a counting chamber. All assays were performed in triplicate.

Plasmid existence and expression in draining lymph nodes (DLNs) and spleen

The quadriceps and anterior tibialis muscles of 6-week-old female BALB/c mice (Hubei Medical Laboratory Animal Center, Wuhan, China) were injected with plasmid pCCL19/ GFP (200 $\mu$ g per mice) or phosphate-buffered saline (PBS) ( $\mathrm{pH}$ 7.2). The DLNs (popliteal and inguinal lymph nodes) and spleens were collected $2 \mathrm{~d}$ after injection. Total DNA was iso- 
lated as previously described ${ }^{[27]}$ and analyzed for the presence of plasmid pCCL19/GFP by PCR. Primers specific for GFP were as follows: sense primer, 5' CGAGCTGTT-3'; antisense primer, 5'-TCACTTGTACAGCTCATCCATGCCGT-3'. The PCR products were analyzed by $1 \%$ agarose gel electrophoresis and DNA sequencing. In the expression experiment, $3 \mathrm{~d}$ after immunization, the spleens and DLNs were harvested, fixed in $4 \%$ paraformaldehyde, embedded in paraffin, and sectioned. The sections were deparaffinized, and the expression of the GFP-labeled recombinant protein was observed under a fluorescent microscope. Additionally, the spleens were minced, passed through a nylon sieve, and treated with RBC lysis buffer (eBiosciences, USA) to remove red blood cells. The DLNs were digested with $0.25 \%$ collagenase IV (Gibco, USA) at $37^{\circ} \mathrm{C}$ for $1 \mathrm{~h}$ with agitation; then, the cells were filtered through a nylon sieve. The percentage of GFP-positive cells was determined using flow cytometry.

\section{Flow cytometric analysis}

The following antibodies obtained from eBiosciences were used for flow cytometric analysis: PE-antimouse CCR7, FITCantimouse CD11c, and PE-antimouse MHCII (I-A ${ }^{b}$ a chain). For staining, the cells were resuspended with PBS containing $2 \%$ BSA and $0.1 \% \mathrm{NaN}_{3}$ at a concentration of $10^{6}-10^{7}$ cells per $100 \mu \mathrm{L}$ and incubated at $4^{\circ} \mathrm{C}$ for $30 \mathrm{~min}$ with appropriately diluted antibodies. After staining, the cells were washed and resuspended in PBS and analyzed on a FACScan analyzer (Becton Dickinson, Mountain View, CA, USA).

\section{Immunization and sample collection}

Six-week-old female BALB/c mice, 5 per group, were injected with $100 \mu \mathrm{g}$ of pCIA-P plus $100 \mu \mathrm{g}$ of chemokine adjuvant plasmids into the quadriceps muscles. To examine the effect of CCL19-encoding plasmid DNA backbone, the mice were co-immunized intramuscularly with $100 \mu \mathrm{g}$ of pCIA-P plus $100 \mu \mathrm{g}$ of the blank pAcGFP1-N1 vector or $100 \mu \mathrm{L}$ PBS $(\mathrm{pH}$ 7.2) in parallel. The mice were treated with pCCL19/GFP, pAcGFP1-N1, or PBS in the quadriceps muscles, followed by pCIA-P immunization at the same vaccination site $24 \mathrm{~h}$ later. All the mice were boosted at weeks 2 and 4; blood samples were collected at 4, 6, 8, 10, 12, 14, and 16 weeks after the first immunization. Peripheral blood was collected from the retroorbital plexus with a syringe and maintained at $4{ }^{\circ} \mathrm{C}$ overnight. Serum was obtained by centrifugation the next day and kept at $-80^{\circ} \mathrm{C}$ until needed.

\section{Antibody analysis}

The levels of specific antibodies against PAc in the serum were determined by an enzyme-linked immunosorbent assay (ELISA). Maxisorp microtiter plates (Corning Costar, Cambridge, MA, USA) were coated with recombinant PAc (10 $\mu \mathrm{g} / \mathrm{mL}$ in carbonate buffer, $\mathrm{pH} 9.6)$ overnight at $4{ }^{\circ} \mathrm{C}$. The plates were blocked with $3 \%$ BSA in PBS containing $0.05 \%$ Tween 20 (PBST) at $37^{\circ} \mathrm{C}$ for $1.5 \mathrm{~h}$ and then overlaid with diluted mice serum samples. Subsequently, the plates were washed and incubated with a peroxidase-conjugated goat antimouse IgG (1:10000, Pierce Biotechnology, Rockford, IL, USA). This was followed by incubation with $o$-phenylenediamine substrate with $\mathrm{H}_{2} \mathrm{O}_{2}$ and then stopped with $2 \mathrm{~mol} / \mathrm{L} \mathrm{H}_{2} \mathrm{SO}_{4}$. The optical density at $490 \mathrm{~nm}\left(O D_{490}\right)$ was recorded. A standard curve was established for each plate, at which point the wells were coated with purified unconjugated goat anti-mouse IgG (Sigma-Aldrich, St Louis, USA), and the mouse reference serum (Bethyl Laboratories, Montgomery, TX, USA) was added in serial dilutions. All samples were tested in duplicate wells. The sample antibody concentration was calculated by a computer program based on multi-parameter logistic algorithms and then interpolated on standard curves.

\section{Cytokine ELISA}

The experiment was conducted to determine the cytokine responses when lymphocytes were restimulated with the similar antigen in vitro. The mice were immunized using the method described above. The immunized mice were euthanized 1 week after the last immunization, and the spleens were isolated and pooled aseptically. Subsequently, the spleen cell suspensions were prepared and cultured in RPMI1640 medium supplemented with $10 \%$ FBS, $1.5 \mathrm{mmol} / \mathrm{L}$ L-glutamine, and $100 \mathrm{U} / \mathrm{mL}$ of penicillin/streptomycin at a concentration of $4 \times 10^{6}$ cells $/ \mathrm{mL}$. One hundred microliters of splenocyte suspension $\left(4 \times 10^{6}\right.$ cells $\left./ \mathrm{mL}\right)$ from each mouse was seeded in triplicate into 96-well flat-bottomed tissue culture plates along with $100 \mu \mathrm{L}$ of complete RPMI-1640 medium containing the mitogen [rPAc protein $(20 \mu \mathrm{g} / \mathrm{mL})]$ or without mitogen (control). The cell cultures were incubated at $37^{\circ} \mathrm{C}$ in a humidified $5 \% \mathrm{CO}_{2}$ environment. After $48 \mathrm{~h}$ of incubation, the plates were centrifuged, and the cell supernatants were harvested. The levels of murine interleukin (IL)-4 and interferon (IFN)- $\gamma$ in the supernatants were measured using commercial cytokine ELISA kits (R\&D Systems, Oxon, UK), according to the manufacturer's instructions. All assays were performed in triplicate.

\section{Statistical analysis}

SPSS 10.0 software (SPSS, Inc, Chicago, IL, USA) was used to perform statistical analyses. The differences in the percentages of migrated DCs, antibody levels and cytokine production among groups were determined by one-way analysis of variance (ANOVA), followed by multiple-mean comparisons using the Student-Newman-Keuls test. $P<0.05$ was considered significant.

\section{Results}

\section{GFP-labeled chemokine plasmid construction and expression}

A plasmid consisting of GFP-labeled CCL19 and pCCL19/ GFP was constructed and tested for protein expression. The murine ccl19 gene was cloned into the pAcGFP1-N1 vector as described in the Materials and methods section (Figure 1A). To confirm whether the CCL19-GFP fusion protein could be expressed by the construct in eukaryotic cells, COS-7 cells were transfected with pCCL19/GFP, and the expression of 
A

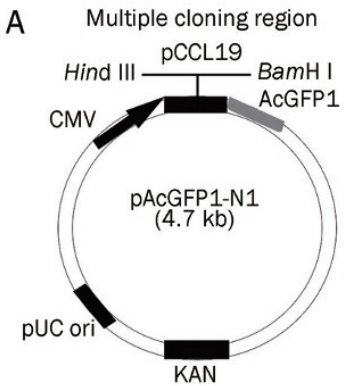

B

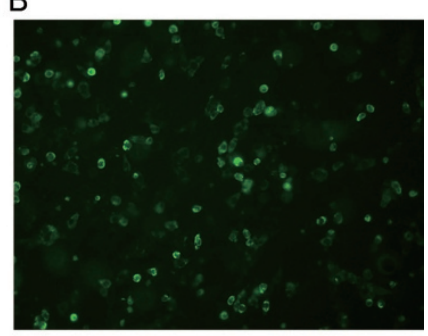

A

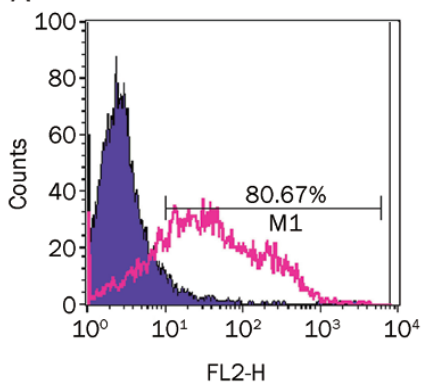

B

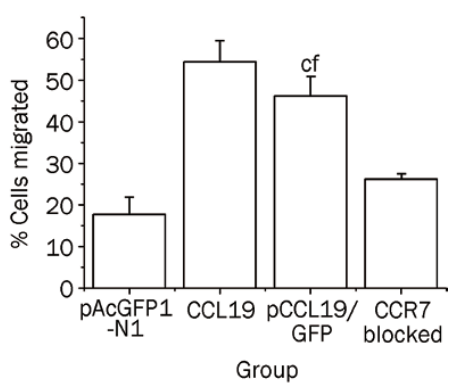

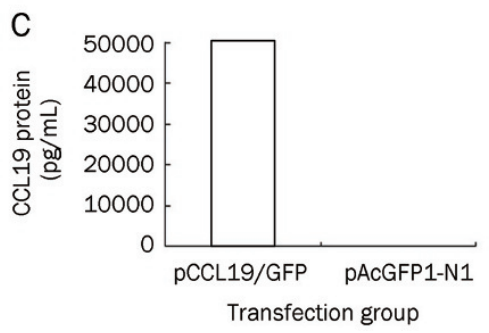

Figure 1. The CCL19-GFP fusion plasmid encodes a functional chemokine. (A) Schematic representation of the chemokine expression plasmid. The ccl19 gene was cloned into the multiple cloning region of the expression vector PAcGFP1-N1 using the restriction enzyme sites, Hind III and BamH I. (B) Transient expression of CCL19-GFP fusion protein in COS-7 cells $(\times 200)$. (C) CCL19 protein is detectable by sandwich ELISA in the supernatant of COS-7 cells transfected with pCCL19/GFP but not with the pAcGFP1-N1 control.

CCL19-GFP fusion protein was detected by fluorescence microscopy and ELISA. Forty-eight hours after transfection, specific green fluorescence could be observed in pCCL19/ GFP transfected cells under a fluorescence microscope (Figure 1B), and cell culture supernatants collected from pCCL19/ GFP transfected cells contained as much as $50,100 \mathrm{pg} / \mathrm{mL}$ of CCL19 fusion protein measured with a mouse CCL19 ELISA kit. The CCL19 concentration in the pAcGFP1-N1 transfected cell culture supernatants was not detectable (Figure 1C).

\section{pCCL19/GFP-expressed fusion protein in vitro chemotactic activity}

We tested whether the fusion of GFP to CCL19 would affect the chemotactic activity of the recombinant protein and whether this chemotactic activity was mediated by CCR7. The supernatants from pCCL19/GFP transfected COS-7 cells were used to analyze the DCs chemotactic activity. Bone marrowderived DCs were stimulated with LPS for $24 \mathrm{~h}$ to increase CCR7 expression on the surface of the DCs. Flow cytometry analysis revealed that approximately $80.67 \%$ of the CD11c ${ }^{+}$ DCs expressed CCR7 after LPS treatment, which was much higher than the expression by the unstimulated cells (data not shown) (Figure 2A). The LPS-stimulated DCs were used in a Transwell system to assess DCs chemotaxis towards CCL19GFP fusion protein. Additionally, another set of DC cultures was incubated with anti-mouse CCR7 antibody before Transwell system application to determine whether the chemotaxis
Figure 2. Chemotactic activity of CCL19 on mature dendritic cells. (A) Bone marrow-derived DCs were generated from BALB/c mice in the presence of GM-CSF. After $7 \mathrm{~d}$ of culture, the cells were harvested and incubated with LPS $(100 \mathrm{ng} / \mathrm{mL})$ for an additional $24 \mathrm{~h}$. CCR7 expression levels in CD11c-positive cells were determined by flow cytometry. DCs were stained with a control isotype antibody (filled histogram) and PEantimouse CCR7 antibody (open histogram). (B) Chemotactic activity of culture medium from COS-7 cells transfected with pCCL19/GFP on mouse bone marrow-derived DCs. DCs $\left(5 \times 10^{5}\right)$ were applied to the upper wells of chemotaxis chambers. The lower wells contained culture supernatants of COS-7 cells transfected with pCCL19/GFP, culture supernatants of COS-7 cells transfected with pAcGFP1-N1, or recombinant mouse CCL19. Additionally, DCs blocked with CCR7 antibody were used in pCCL19/GFP group. Four hours later, the cells migrating to the bottom chamber were counted. Mean \pm SD. $n=3 .{ }^{c} P<0.01$ vs the pAcGFP1-transfected group, ${ }^{\mathrm{f}} P<0.01$ vs the CCR7 blocked group.

was due to the ligand-receptor interaction between CCL19 and CCR7. As shown in Figure 2B, the DCs were strongly attracted to the culture supernatants from pCCL19/GFP-transfected cells, and the chemotaxis was clearly blocked by the CCR7 antibody $(P<0.01)$. These results indicated that CCL19 could properly exert its chemotactic function in a GFP fusion form. Furthermore, chemokine-driven chemotaxis of mature DCs was primarily mediated by CCR7.

\section{Specific anti-PAc antibody responses in mice}

Experiments were performed to compare the serum PAcspecific IgG levels in mice intramuscularly immunized with pCIA-P to mice immunized with pCIA-P plus pCCL19/GFP (Figure 3). An increase in the serum IgG anti-PAc antibody response was observed in the coadministered group throughout the entire experimental period, which was significantly different from the control group at weeks $4-14(P<0.01$ or $P<0.05)$. The data analysis demonstrated that the DNA plasmid expressing CCL19 enhanced serum PAc-specific IgG antibody responses when it was used in combination with anticaries DNA vaccine pCIA-P.

\section{Cytokine secretion by restimulated splenocytes}

We used quantitative ELISA to examine the effect of coadministering pCCL19/GFP on the Th1- and Th2-type cytokines produced by the restimulated splenocytes. BALB/c mice, immunized intramuscularly on three occasions, were sacrificed 1 week after the last immunization to obtain spleen cells. 


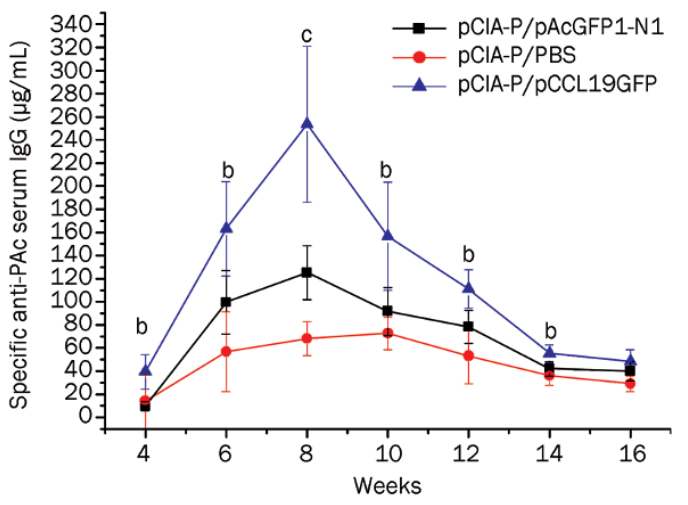

Figure 3. Serum PAc-specific IgG antibody levels. BALB/c mice groups $(n=5)$ were immunized intramuscularly on three occasions at 7 -d intervals. Serum samples were collected at 4, 6, 8, 10, 12, 14, and 16 weeks after the first immunization. Specific anti-PAc serum IgG concentration was determined by ELISA. Mean \pm SD. $n=5$. ${ }^{\mathrm{b}} P<0.05,{ }^{\mathrm{c}} P<0.01$ vs the vector control group. (घ) the group immunized with pCIA-P and pAcGFP1-N1; $(\bullet)$ the group immunized with pCIA-P and PBS; $(\boldsymbol{\Delta})$ the group immunized with pCIA-P and PCCL19/GFP.

The splenocytes were then stimulated with PAc protein. As shown in Figure 4, there was no significant IFN-ץ or IL-4 production detected in the splenocytes of mice that received the control vector (pCI-neo) after restimulation with the PAc protein. By comparison, the splenocytes of all the groups vaccinated with PCIA-P produced both IFN-Y and IL-4 at different levels. The mice that were co-immunized with pCCL19/GFP demonstrated significantly higher IFN-ץ levels compared with
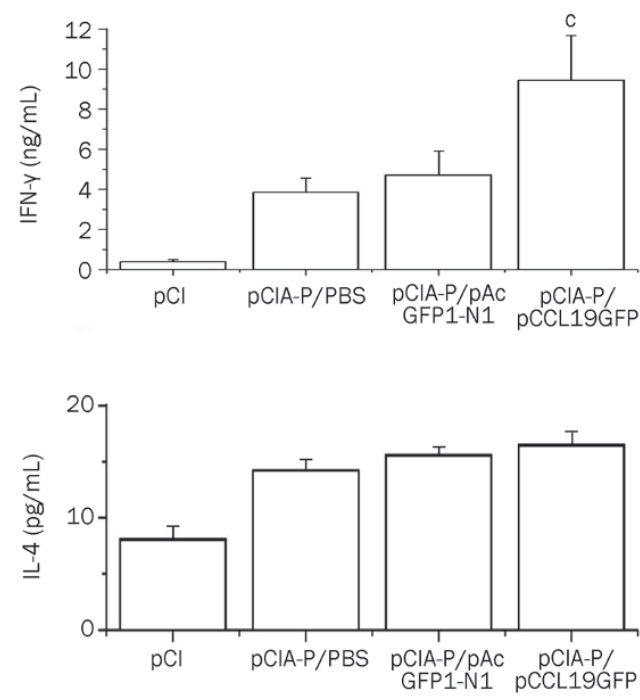

Figure 4. Cytokine production by antigen-restimulated splenocytes. The splenocytes were generated from groups of immunized mice and restimulated with PAc protein. IFN-y and IL-4 concentrations in cell culture supernatants were assessed by ELISA method. The results are shown as the mean \pm standard deviation of three independent experiments. Mean \pm SD. $n=3 .{ }^{c} P<0.01$ vs the group immunized with $\mathrm{pCIA}-\mathrm{P}$ and pACGFP1-N1. the other groups $(P<0.01)$, whereas, there was no significant increase in the IL-4 production observed in the coadministered group.

\section{Presence and expression of pCCL19/GFP in spleen and DLNs}

We hypothesized that the increased immune responses in CCL19 DNA-treated mice might be caused by the exogenous CCL19 expression and the subsequent DC recruitment in the secondary lymphoid tissues; therefore, we first studied the presence and expression of pCCL19/GFP in the spleen and DLNs after intramuscular immunization. Plasmid-containing gfp gene detection is an appropriate method to rule out possible interference due to endogenous ccl19 gene expression. In the present study, lymphoid tissue DNA extracts from normal mice were spiked with pCCL19/GFP and used as positive controls. DNA extracts from PBS-treated mice were used as negative controls. The PCR analysis demonstrated that pCCL19/GFP could be detected in the spleens and DLNs 48 $\mathrm{h}$ after injection (Figure 5). In the end, a 720-bp PCR product was amplified, and DNA sequencing confirmed that the nucleotide sequence of the amplified product was identical to that of the $g f p$ gene. No PCR product was obtained from either the spleens or the DLNs of the PBS-treated mice. Subsequently, we assessed pCCL19/GFP expression in the spleen and DLNs. As shown in Figure 6, specific green fluorescence could be observed in the pCCL19/GFP-treated mice tissues. The percentage of cells expressing pCCL19/GFP in the spleen and DLNs was analyzed using flow cytometry. Approximately $5.1 \%$ and $7.99 \%$ GFP-positive cells were detected in spleen and DLNs of pCCL19/GFP-treated mice, respectively (Figure 7).

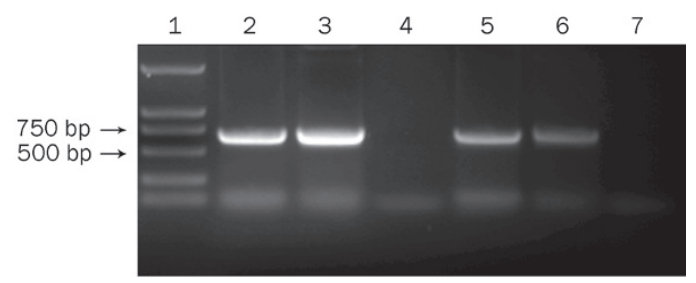

Figure 5. PCR analysis of plasmid existence in mouse spleen and the DLNs (popliteal and inguinal lymph nodes) $48 \mathrm{~h}$ after intramuscular delivery of plasmids. Lane 1, DNA marker DL2000; Lane 2, the spleen DNA samples from pCCL19/GFP-immunized mice; Lane 3, the spleen DNA samples from normal mice spiked with pCCL19/GFP; Lane 4, the spleen DNA samples from PBS-immunized mice; Lane 5, the DLNs DNA samples from normal mice spiked with pCCL19/GFP; Lane 6, the DLNs DNA samples from pCCL19/GFP-immunized mice; and Lane 7, the DLNs DNA samples from PBS-immunized mice.

pCCL19/GFP-expressed chemokine protein in vivo chemotactic activity

To determine whether the elevated immune response in pCCL19/GFP-injected mice occurred as a result of increased DC-T cell interaction in secondary lymphoid tissues, the number of mature DCs $\left(\mathrm{CD} 11 \mathrm{c}^{+}\right.$major histocompatibility complex 


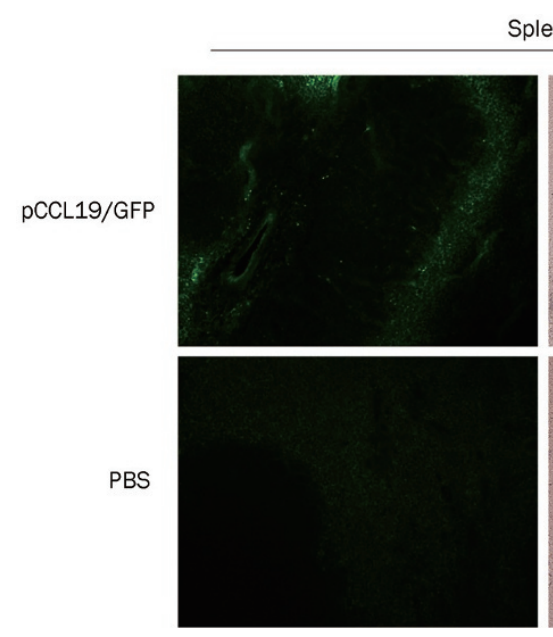

Spleen
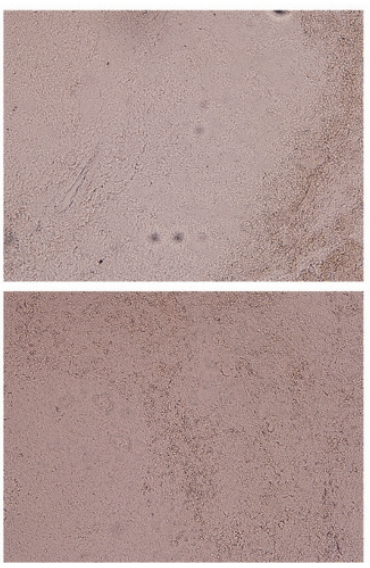

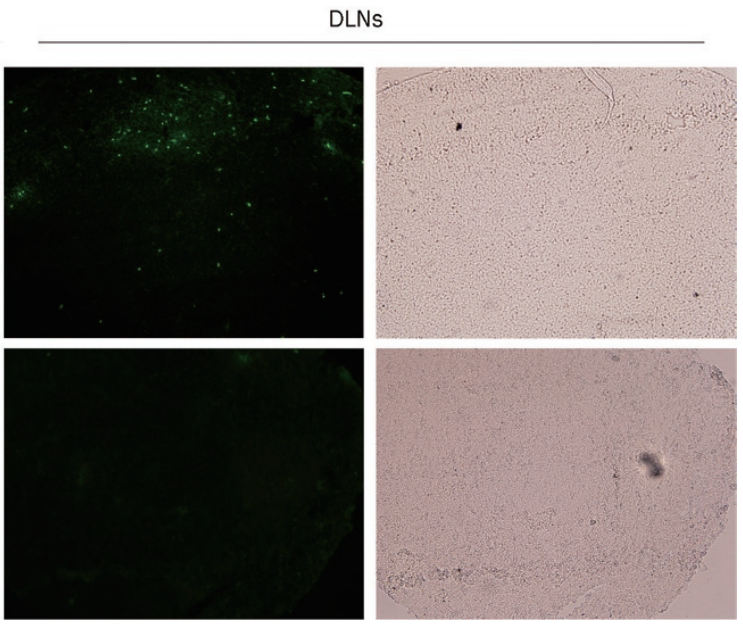

Figure 6. CCL19-GFP fusion protein expression in secondary lymphoid tissues analyzed under the microscope. DLNs and spleens from mice injected intramuscularly with pCCL19/GFP or PBS (negative control) were harvested $3 \mathrm{~d}$ after immunization. Sections were made from these tissues. GFP fluorescence was observed under a fluorescence microscope. Bright field images in the same site were also provided. All slides represent original magnification $(200 \times)$.
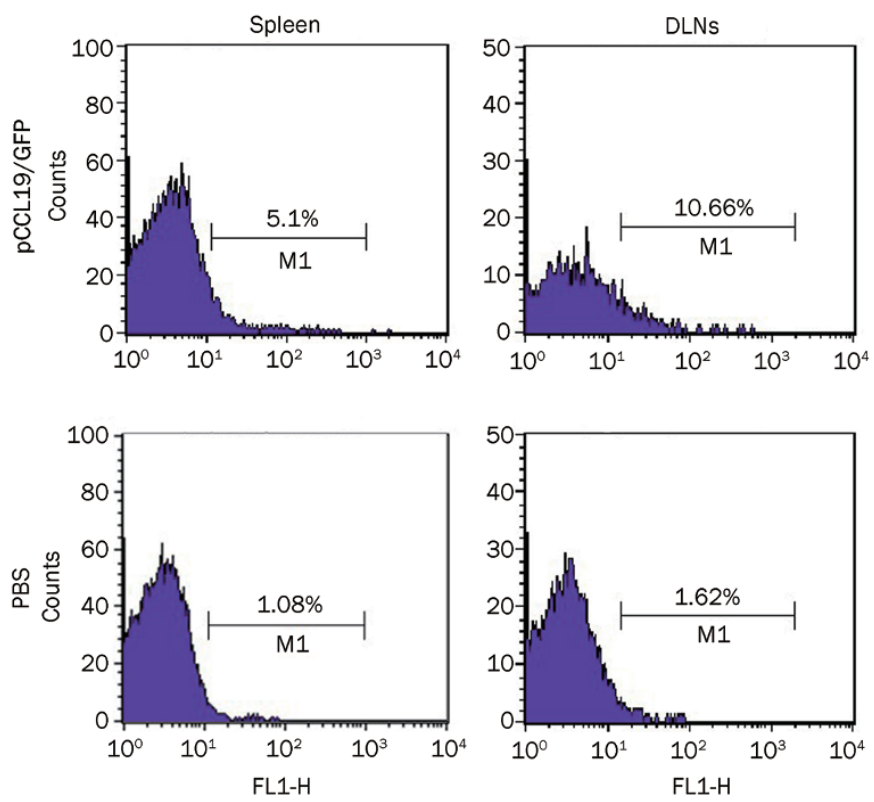

Figure 7. CCL19-GFP fusion protein expression in secondary lymphoid tissues analyzed by flow cytometry. Splenocytes and DLNs cells were prepared from pCCL19/GFP- or PBS-treated mice $3 \mathrm{~d}$ after immunization. The percentage of GFP-positive cells was detected. This result is representative of three experiments.

class II ${ }^{\text {high }}$ ) was measured in the spleen and DLNs. Three days after intramuscular administration, the mice treated with CCL19 DNA showed increased size and total cell numbers in their DLNs and spleens compared with mice treated with control vector (data not shown). Moreover, as shown in Figure 8, the splenocytes and DLN cells isolated from mice that received CCL19 DNA exhibited a higher percentage and number of CD11 $\mathrm{c}^{+}$major histocompatibility complex class $\mathrm{II}^{\mathrm{high}}(\mathrm{MHC}$

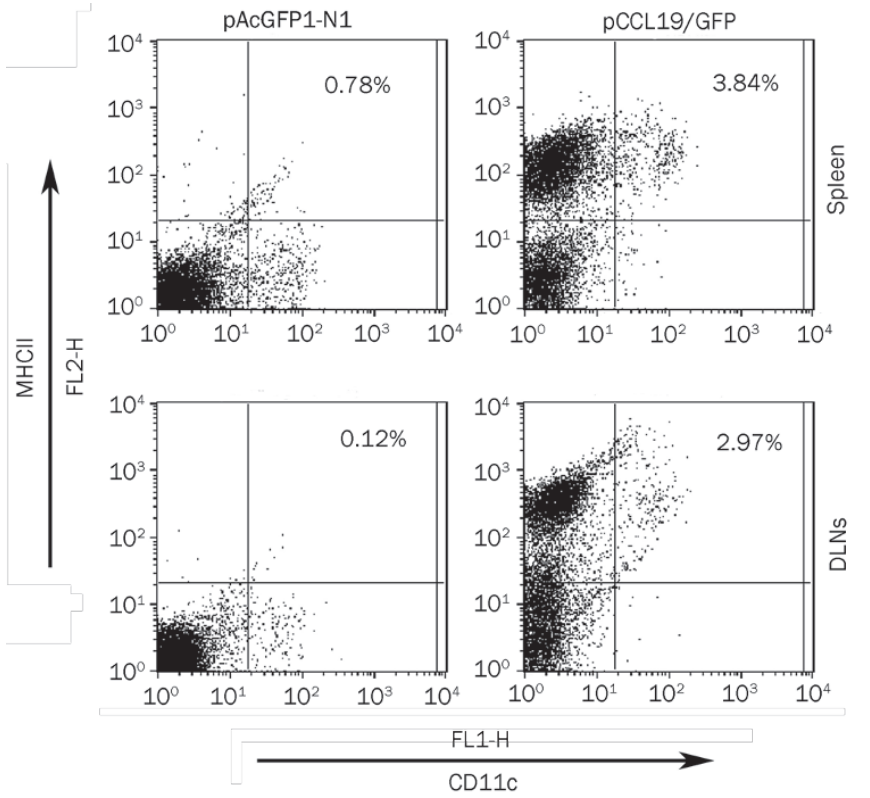

Figure 8. CCL19 increases the number of mature DCs in secondary lymphoid tissues. Splenocytes and DLN cells were prepared from pAcGFP1-N1- or pCCL19/GFP-treated mice using collagenase digestion $3 \mathrm{~d}$ after immunization and stained for DC marker (CD11c) and class II marker (I-A $\alpha$-chain), followed by flow cytometry analysis. This result is representative of three experiments.

$\mathrm{II}^{\text {high }}$ ) cells than the control injected mice. While the control vector-treated mice displayed $0.78 \%$ of $\mathrm{CD} 11 \mathrm{c}^{+} \mathrm{MHC} \mathrm{II}^{\text {high }}$ cells in the spleen and $0.12 \%$ in DLNs, CCL19-treated mice expressed $3.84 \%$ and $2.97 \%$ in the spleen and DLNs, respectively. Taken together, CCL19 DNA administration increases the number of mature DCs in secondary lymphoid tissues. 


\section{Discussion}

DCs are APCs that localize at peripheral tissues and migrate into the $\mathrm{T}$ cell areas of secondary lymphoid organs to present antigens to $\mathrm{T}$ cells. DC migration is thought to be one of the most important events for the induction of protective immunity against pathogens. Chemokines are small proteins $(6-14 \mathrm{kDa})$ that act primarily as chemoattractants for specific types of leukocytes and play important roles in inflammatory responses and the formation and organization of lymphoid organs. Recent studies have indicated that chemokines can act as adjuvants to DNA vaccination via the recruitment of DCs and other immune cells to immune inductive sites ${ }^{[20-23,28]}$. Specifically, CCL19 has been reported to exert a critical function in the initiation of immune responses owing to its chemotactic effect on CCR7-expressing mature DCs and naive T/B cells ${ }^{[29-31]}$. It is surmised that exogenous CCL19 overexpression in lymphoid tissue may enhance the recruitment of antigenpresenting DCs, thus facilitating the contact between DCs and responder $\mathrm{T}$ cells ${ }^{[2,21,23]}$. In this study, we provided the direct evidence that CCL19 DNA plasmid entered secondary lymphoid tissues and expressed the chemokine after intramuscular administration. Intramuscular injection is a traditional and widely used method for DNA inoculation, which has been proven to result in plasmid dissemination and protein expression in DLNs as well as at distal sites in lymphoid and nonlymphoid tissues for a couple of days ${ }^{[32-34]}$. However, the mechanisms of plasmid DNA uptake and distribution are still not completely clear. Following intramuscular inoculation, antigen-presenting cells could be transfected by plasmid DNA directly or uptake the antigen from transfected muscle cells. Subsequently, DNA rapidly leaves the site of injection and enters the blood stream or circulates throughout the lymphatic system, both as free DNA and in cells (most likely DCs) that have taken up the plasmid ${ }^{[35]}$. The presence of protein expression at secondary lymphoid organs might result from direct transfection by plasmid DNA at such sites or as a result of seeding by plasmid-transfected cells at inoculation sites. Although we did not demonstrate the exact secondary lymphoid tissue anatomical site for CCL19 DNA plasmid expression in this study, our results indicate that muscular pCCL19/ GFP delivery leads to dissemination of plasmid DNA to secondary lymphoid tissues and that these sites may serve as a source of CCL19 production.

Next, we assessed whether exogenous CCL19 overexpression in secondary lymphoid tissues could improve DC migration to these tissues. CCL19 is a high-affinity functional ligand for CCR7, the receptor molecule expressed on mature $\mathrm{DCs}^{[8,9]}$. It has been demonstrated that CCL19 and CCR7 play an important role in DC homing to lymphoid tissues ${ }^{[15-19]}$. Our findings demonstrate that CCL19-GFP is a potent chemoattractant for in vitro-expanded bone marrow-derived DCs. The specificity was confirmed by CCR7 blockade on mature DCs. Other researchers also reported that not only the stably transfected CCR7 cells but also the endogenously expressing CCR7 cells responded to CCL19 by cell migration ${ }^{[36]}$. Additionally, our study revealed that the spleens and DLNs of mice sys- temically injected with CCL19 DNA had a significant increase in the number of mature DCs, which orchestrated more efficient induction of immune responses. Other researchers have investigated the DCs signaling module of chemotaxis function by CCL19 and CCR7. They observed that chemotaxis was regulated by the pertussis toxin-sensitive Gi proteins as well as the three MAPK family members: Erk1/2, p38, and JNK ${ }^{[37]}$.

Improved DC migration in CCL19 DNA recipients provides an increased frequency of encounters with naive $\mathrm{T}$ cells. Using these findings as a proof of concept, we observed a significant increase in the serum PAc-specific IgG level compared with the vector DNA-treated control, which corroborates the previous observation. Furthermore, an enhanced ability of the restimulated spleen cells to produce IFN- $\gamma$ was observed in the pCCL19/GFP co-immunized mice compared with vector DNA co-immunized mice, indicating a Th1 shift in the presence of pCCL19/GFP. Following CCL19 DNA co-immunization, enhanced $\mathrm{T}$ cell responses were observed to differ in nature. Codelivery of CCL19 DNA with an anti-herpes simplex virus DNA vaccine enhanced the production of both Th1- and Th2type cytokines by $\mathrm{CD} 4^{+} \mathrm{Th}$ cells ${ }^{[20]}$. In other studies, coadministration of CCL19 DNA resulted in the enhancement of a Th1polarized immune response to tumor antigen ${ }^{[2,25]}$ and pseudorabies virus ${ }^{[23]}$. The cause of this difference is unclear. One possible reason might be due to the different immunogenicity of antigens. Our previous studies found that animals immunized with anti-caries DNA vaccine (pCIA-P or pGJA-P/VAX) tended to exhibit augmented IFN- $\gamma$ cytokine levels secreted by $\mathrm{CD}^{+}{ }^{+} \mathrm{Th}$ cells ${ }^{[38,39]}$. The second reason might be related to the routes or methods of DNA immunization. Some reports indicate that mice receiving intramuscular DNA inoculations predominantly produce Th1 responses ${ }^{[40,41]}$. Additionally, the DNA vaccine itself induces a preferential Th1 response ${ }^{[42,43]}$.

In conclusion, our study demonstrated that codelivery of the CCL19 gene with an anti-caries DNA vaccine pCIA-P via intramuscular injection significantly augmented systemic immune responses to pCIA-P as well as IFN- $\gamma$ production in mice compared with the pCIA-P vaccination. We also detected the presence and expression of GFP labeled CCL19 plasmid in the spleen and DLNs, which might explain the increased number of mature DCs in these tissues and the enhanced immunogenicity of the DNA vaccine. Our results demonstrate that CCL19 is an attractive adjuvant for the anti-caries DNA vaccine; however, it remains to be seen if further improvements could be achieved. Alternative modulators coadministered with CCL19 or the expression of CCL19 fused with an antigen might provide a more effective vaccine. Additional studies investigating this design are currently being conducted.

\section{Acknowledgements}

This study was supported by grants from the National Natural Science Foundation of China (№ 81000435), the Doctoral Fund of the Ministry of Education of China (№ 200804861011), and the PhD Candidates Self-research (including 1+4) Program of Wuhan University in 2010 (№ 2010304010100163). 


\section{Author contribution}

Ming-wen FAN and Qing-an XU designed the study and revised the manuscript; Yan-hong YAN, Sheng-cai QI, and Ling-kai SU performed the study; and Yan-hong YAN wrote the manuscript.

\section{References}

1 Donnelly JJ, Wahren B, Liu MA. DNA vaccines: progress and challenges. J Immunol 2005; 175: 633-9.

2 Kutzler MA, Weiner DB. DNA vaccines: ready for prime time? Nat Rev Genet 2008; 9: 776-88.

3 Fan MW, Bian Z, Peng ZX, Zhong Y, Chen Z, Peng B, et al. A DNA vaccine encoding a cell-surface protein antigen of Streptococcus mutans protects gnotobiotic rats from caries. J Dent Res 2002; 81: 784-7.

4 Wolff JA, Malone RW, Williams P, Chong W, Acsadi G, Jani A, et al. Direct gene transfer into mouse muscle in vivo. Science 1990; 247 : 1465-8.

5 Porgador A, Irvine KR, Iwasaki A, Barber BH, Restifo NP, Germain RN. Predominant role for directly transfected dendritic cells in antigen presentation to $\mathrm{CD} 8^{+} \mathrm{T}$ cells after gene gun immunization. J Exp Med 1998; 188: 1075-82.

6 Bot A, Stan AC, Inaba K, Steinman R, Bona C. Dendritic cells at a DNA vaccination site express the encoded influenza nucleoprotein and prime $\mathrm{MHC}$ class I-restricted cytolytic lymphocytes upon adoptive transfer. Int Immunol 2000; 12: 825-32.

7 Akbari O, Panjwani N, Garcia S, Tascon R, Lowrie D, Stockinger B. DNA vaccination: transfection and activation of dendritic cells as key events for immunity. J Exp Med 1999; 189: 169-78.

8 Condon C, Watkins SC, Celluzzi CM, Thompson K, Falo LD Jr. DNAbased immunization by in vivo transfection of dendritic cells. Nat Med 1996; 2: 1122-8.

9 Mitoma M, Oho T, Michibata N, Okano K, Nakano Y, Fukuyama M, et al. Passive immunization with bovine milk containing antibodies to a cell surface protein antigen-glucosyltransferase fusion protein protects rats against dental caries. Infect Immun 2002; 70: 2721-4.

10 Dieu MC, Vanbervliet B, Vicari A, Bridon JM, Oldham E, Aït-Yahia S, et al. Selective recruitment of immature and mature dendritic cells by distinct chemokines expressed in different anatomic sites. J Exp Med 1998; 188: 373-86.

11 Sallusto F, Lanzavecchia A. Mobilizing dendritic cells for tolerance, priming, and chronic inflammation. J Exp Med 1999; 189: 611-4.

12 Scheerlinck JY. Genetic adjuvants for DNA vaccines. Vaccine 2001; 19: 2647-56.

13 Zlotnik A, Yoshie O. Chemokines: a new classification system and their role in immunity. Immunity 2000; 12: 121-7.

14 Campbell JJ, Butcher EC. Chemokines in tissue-specific and microenvironment-specific lymphocyte homing. Curr Opin Immunol 2000; 12: $336-41$.

15 Förster R, Schubel A, Breitfeld D, Kremmer E, Renner-Müller I, Wolf E, et al. CCR7 coordinates the primary immune response by establishing functional microenvironments in secondary lymphoid organs. Cell 1999; 99: 23-33.

16 Cyster JG. Chemokines and cell migration in secondary lymphoid organs. Science 1999; 286: 2098-102.

17 Zlotnik A, Morales J, Hedrick JA. Recent advances in chemokines and chemokine receptors. Crit Rev Immunol 1999; 19: 1-47.

18 Sallusto F, Lanzavecchia A. Understanding dendritic cell and T-lymphocyte traffic through the analysis of chemokine receptor expression. Immunol Rev 2000; 177: 134-40.
19 Luther SA, Tang HL, Hyman PL, Farr AG, Cyster JG. Coexpression of the chemokines ELC and SLC by T zone stromal cells and deletion of the ELC gene in the plt/plt mouse. Proc Natl Acad Sci U S A 2000; 97: 12694-9.

20 Eo SK, Lee S, Kumaraguru U, Rouse BT. Immunopotentiation of DNA vaccine against herpes simplex virus via co-delivery of plasmid DNA expressing CCR7 ligands. Vaccine 2001; 19: 4685-93.

21 Lee Y, Eo SK, Rouse RJ, Rouse BT. Influence of CCR7 ligand DNA preexposure on the magnitude and duration of immunity. Virology 2003; 312: 169-80.

22 Toka FN, Gierynska M, Rouse BT. Codelivery of CCR7 ligands as molecular adjuvants enhances the protective immune response against herpes simplex virus type 1. J Virol 2003; 77: 12742-52.

23 Han YW, Aleyas AG, George JA, Kim SJ, Kim HK, Yoo DJ, et al. Genetic co-transfer of CCR7 ligands enhances immunity and prolongs survival against virulent challenge of pseudorabies virus. Immunol Cell Biol 2009; 87: 91-9.

24 Westermann J, Nguyen-Hoai T, Baldenhofer G, Höpken UE, Lipp M, Dörken B, et al. CCL19 (ELC) as an adjuvant for DNA vaccination: induction of a TH1-type T-cell response and enhancement of antitumor immunity. Cancer Gene Ther 2007; 14: 523-32.

25 Nguyen-Hoai T, Baldenhofer G, Ahmed MS, Pham-Duc M, Gries M, Lipp $M$, et al. CCL19 (ELC) improves TH1-polarized immune responses and protective immunity in a murine Her2/neu DNA vaccination model. J Gene Med 2012; 14: 128-37.

26 Xu Q, Katz J, Zhang P, Ashtekar AR, Gaddis DE, Fan M, et al. Contribution of a Streptococcus mutans antigen expressed by a Salmonella vector vaccine in dendritic cell activation. Infect Immun 2011; 79 : 3792-800.

27 Xu QA, Yu F, Fan MW, Bian Z, Chen Z, Fan B, et al. Immunogenicity and persistence of a targeted anti-caries DNA vaccine. J Dent Res 2006; 85: 915-8.

28 Kutzler MA, Weiner DB. Developing DNA vaccines that call to dendritic cells. J Clin Invest 2004; 114: 1241-4.

29 Banchereau J, Steinman RM. Dendritic cells and the control of immunity. Nature 1998; 392: 245-52.

30 Mellman I, Steinman RM. Dendritic cells: specialized and regulated antigen processing machines. Cell 2001; 106: 255-8.

31 Wen H, Schaller MA, Dou Y, Hogaboam CM, Kunkel SL. Dendritic cells at the interface of innate and acquired immunity: the role for epigenetic changes. J Leukoc Biol 2008; 83: 439-46.

32 Tuomela M, Malm M, Wallen M, Stanescu I, Krohn K, Peterson P. Biodistribution and general safety of a naked DNA plasmid, GTUMultiHIV, in a rat, using a quantitative PCR method. Vaccine 2005; 23: 890-6.

33 Coelho-Castelo AA, Trombone AP, Rosada RS, Santos RR Jr, Bonato VL, Sartori A, et al. Tissue distribution of a plasmid DNA encoding Hsp65 gene is dependent on the dose administered through intramuscular delivery. Genet Vaccines Ther 2006; 4: 1.

34 Liu C, Fan M, Xu Q, Li Y. Biodistribution and expression of targeted fusion anti-caries DNA vaccine PGJA-P/VAX in mice. J Gene Med 2008; 10: 298-305.

35 Iwasaki A, Torres CAT, Ohashi PS, Robinson HL, Barber BH. The dominant role of bone marrow-derived cells in CTL induction following plasmid DNA immunization at different sites. J Immunol 1997; 159: 11-4.

36 Yoshida R, Imai T, Hieshima K, Kusuda J, Baba M, Kitaura M, et al. Molecular cloning of a novel human CC chemokine EBI1-ligand chemokine that is a specific functional ligand for EBI1, CCR7. J Biol Chem 1997; 272: 13803-9.

37 Sánchez-Sánchez N, Riol-Blanco L, Rodríguez-Fernández JL. The 
multiple personalities of the chemokine receptor CCR7 in dendritic cells. J Immunol 2006; 176: 5153-9.

38 Liu GX, Xu QA, Jin J, Li YH, Jia R, Guo JH, et al. Mucosal and systemic immunization with targeted fusion anti-caries DNA plasmid in young rats. Vaccine 2009; 27: 2940-7.

39 Li YH, Huang S, Du M, Bian Z, Chen Z, Fan MW. Immunogenic characterization and protection against Streptococcus mutans infection induced by intranasal DNA prime-protein boost immunization. Vaccine 2010; 28: 5370-6.

40 Pertmer TM, Roberts TR, Haynes JR. Influenza virus nucleoproteinspecific immunoglobulin $G$ subclass and cytokine responses elicited by DNA vaccination are dependent on the route of vector DNA delivery. J Virol 1996; 70: 6119-25.

41 Feltquate DM, Heaney S, Webster RG, Robinson HL. Different T helper cell types and antibody isotypes generated by saline and gene gun DNA immunization. J Immunol 1997; 158: 2278-84.

42 Navia JM. Animalmodels in dental research. Tuscaloosa: University of Alabama Press; 1977. p 280.

43 Wang S, Heilman D, Liu F, Giehl T, Joshi S, Huang X, et al. A DNA vaccine producing LcrV antigen in oligomers is effective in protecting mice from lethal mucosal challenge of plague. Vaccine 2004; 22 : 3348-57. 